ORIGINAL BREVE

\title{
Estado situacional de la diabetes mellitus tipo 2 en pacientes adultos mayores en el hospital Santa María del Socorro. Ica, Perú
}

\author{
Esthefany Esbelia Ramos-Mayta ${ }^{\text {1,a }}$, María Candelaria Gonzales-Olarte ${ }^{1, a}$
}

\author{
Facultad de medicina, Universidad Nacional San Luisa Gonzaga. Ica, Perú ${ }^{1}$ \\ Médico cirujano ${ }^{a}$
}

C on el objetivo de determinar las características de la diabetes mellitus tipo 2 en el adulto mayor en el Hospital Santa María del Socorro de Ica en el 2013, se desarrolló un estudio descriptivo, la población estuvo conformada por pacientes adultos mayores con diagnóstico de Diabetes Mellitus tipo 2 atendidos en el Hospital Santa María del Socorro en el 2013. Nuestros hallazgos sugieren que los pacientes de 60 a 75 años de edad (60\%), pacientes de zona urbana (45\%), con grado de instrucción primaria $(36,7 \%)$ y pacientes casados $(38,3 \%)$ son los que presentaron en mayor proporción la Diabetes Mellitus tipo 2, además las características clínicas más frecuentes fueron la infección urinaria; la enfermedad cerebro vascular, y la hipertensión arterial. Finalmente concluimos que existen muchos características sociodemográficas y clínicas de importancia en el manejo del paciente adulto mayor con diabetes mellitus tipo 2.

Palabras clave: Diabetes Mellitus tipo 2, adulto Mayor, características de la población, complicaciones de la Diabetes (fuente: DeCS BIREME).

Autor corresponsal: Esthefany Esbelia Ramos Mayta . Teléfono: (51) 956390861 . Correo electrónico: stfany_r.m@hotmail.com

\section{INTRODUCCIÓN}

El término diabetes mellitus (DM) describe los adultos mayores en el Hospital Santa un desorden metabólico de múltiples etiologías, caracterizado por hiperglucemia crónica con disturbios en el metabolismo de los carbohidratos, grasas y proteínas y que resulta de defectos en la secreción y/o en la acción de la insulina y que afecta a diversos grupos etarios, desde niños y jóvenes, adultos y adultos mayores; tanto en hombres como en mujeres (1).

Además es una enfermedad que va en aumento a nivel mundial causando múltiples estragos en nuestro organismo. Se han realizado muchos estudios sobre la DM, dentro de los cuales se observaron las características demográficas y epidemiológicas, así como factores de riesgo asociados. En nuestro medio la DM tipo 2 ocupa un lugar importante en cuanto a la morbimortalidad; sin embargo aún no se realizan estudios en cuanto a su afectación en el adulto mayor en nuestro departamento; por tal motivo el objetivo de nuestro trabajo fue realizar un análisis situacional de esta enfermedad en
María del Socorro de Ica en el 2013.

\section{MATERIALES Y MÉTODOS}

Realizamos un estudio de tipo descriptivo y observacional. Revisamos las historias clínicas de los pacientes adultos mayores con diagnóstico recién establecido (debut) o previamente establecido de DM tipo 2 que han sido atendidos (Hospitalizados) en el Hospital Santa María del Socorro de Ica del 01 de Enero al 31 de Diciembre del 2013.

Por el tipo de trabajo y características de la población se realizó un muestro no probabilístico, se incluyeron las historias clínicas de los pacientes de 60 a más años, que fueron hospitalizados en el servicio de medicina del Hospital Santa María del Socorro del 01 de Enero al 31 de diciembre del 2013, a quienes se les diagnosticó DM tipo 2 durante el transcurso de su hospitalización o debut (desde el ingreso hasta el alta) y/o que tuvieron diagnostico establecido de Diabetes Mellitus tipo 2
Se excluyeron aquellas historias que no tuvieron diagnostico establecido de Diabetes Mellitus tipo 2 y a quienes no les diagnosticaron DM tipo II durante el transcurso de su hospitalización y/o cuyas Historias clínicas se encontraron incompletas o con datos insuficientes $y / 0$ que tuvieron un diagnostico dudoso de DM tipo II y/o que fueron atendidos fuera del tiempo de estudio establecido.

Los datos obtenidos de la revisión de historias cínicas fueron ingresados a una base de datos utilizando el software Microsoft Excel en su versión 2010. El procesamiento y análisis de los datos se realizó mediante el programa estadístico SPSS (por sus siglas en inglés: Statistical Package of the Social Sciences), en su versión 18.0 para Windows. Se utilizaron medidas de estadística descriptiva. Se realizó el análisis de las variables utilizando tablas de distribución de frecuencias para las variables nominales.

Los datos se recogieron directamente de las historias clínicas del paciente las mismas que estuvieron disponibles en el servicio de hospitalización durante la estadía del paciente. Se identificaron las variables a ser estudiadas en las historias clínicas y fueron recolectadas en las respectivas fichas.

\section{RESULTADOS}

CARACTERÍSTICAS SOCIODEMOGRAFICAS DE LA POBLACION EN ESTUDIO.

En la Tabla 1 se describen las principales características sociodemográficas de la población en estudio, notamos que el grupo etario de 60 a 75 años, el sexo femenino, la procedencia urbana y el grado de instrucción primaria, son los que tienen mayor frecuencia de sufrir DM tipo 2. 


\begin{tabular}{llcc}
\hline \multirow{2}{*}{ TABLA 1 } & \multicolumn{3}{c}{ Características sociodemográficas de los pacientes adultos mayores con Diabetes } \\
& Mellitus tipo 2, hospitalizados en el Hospital Santa María del Socorro. Ica, Perú 2013 \\
\hline \multirow{4}{*}{ Características } & N=60 & $\%$ \\
\hline \multirow{3}{*}{ Sexo } & 60 a 75 años & 36 & 60,0 \\
& 76 a más años & 24 & 40,0 \\
\multirow{3}{*}{ Procedencia } & Masculino & 23 & 38,3 \\
& Femenino & 37 & 61,7 \\
& Urbano & 27 & 45,0 \\
Grado de instrucción & Rural & 10 & 16,7 \\
& Urbano-marginal & 23 & 38,3 \\
& Sin estudios & 13 & 21,7 \\
& Primaria & 22 & 36,7 \\
& Secundaria & 16 & 26,7 \\
& Superior & 9 & 15,0 \\
\hline
\end{tabular}

Características clínicas de los pacientes adultos mayores con Diabetes Mellitus tipo 2, hospitalizados en el Hospital Santa María del Socorro. Ica, Perú 2013

\begin{tabular}{llcc} 
& \multicolumn{1}{c}{ Características } & $\mathbf{N}=\mathbf{6 0}$ & \% \\
\hline Causa de descompensación & Infección & 46 & 76,7 \\
& Problema cardiovascular & 6 & 10,0 \\
& Problema metabólico & 3 & 5,0 \\
& Problema renal & 3 & 5,0 \\
& Otros & 2 & 3,3 \\
Tiempo de hospitalización & 1 a 5 días & 41 & 68,3 \\
& 11 a 15 días & 17 & 28,3 \\
Diagnóstico & 15 a más días & 2 & 3,3 \\
& Debut & 4 & 6,7 \\
& 1 a 5 años & 15 & 25,0 \\
& 6 a 10 años & 30 & 50,0 \\
& 11 a 15 años & 3 & 5,0 \\
& Más de 15 años & 8 & 13,3 \\
\hline
\end{tabular}

CARACTERÍSTICAS CLÍNICAS DE LA POBLACIÓN EN ESTUDIO.

En la tabla 2 se detallan las principales características clínicas de la población en estudio, la principal causa de descompensación fue por infección, el tiempo de hospitalización fue mayormente entre 1 a 5 días, el diagnóstico de la enfermedad se dio luego de 6 a 10 años en mayor proporción.

En la figura 1, se describe los principales tipos de infecciones según área de afectación, notamos que la infección urinaria fue el tipo más frecuente.

\section{DISCUSIÓN}

La frecuencia de pacientes diagnosticados precozmente (debut) hallada en el estudio fue de $6,7 \%$, esta cifra es significativamente menor a la publicada por Brenes (2), quien refiere una frecuencia de $79 \%$ en el estudio realizado en Costa Rica.

Se ha hallado que la causa que motivó la hospitalización en la población de estudio fueron las infecciones alcanzando un $76,7 \%$ del total de las causas, mientras que los problemas cardiovasculares solo alcanzan un $10 \%$, este resultado se diferencia de los referidos por De la Paz (3), quien menciona en su estudio como primera causa de hospitalización a los problemas cardiovasculares, siendo la más frecuentes la hipertensión arterial con un $36 \%$.

En nuestro estudio, se ha identificado que el grado de instrucción más frecuente fue primaria, con un $36,7 \%$, esta cifra es significativamente menor a la publicada por Tapia (4), quien reporta un $60 \%$ correspondiente a los analfabetos y primaria completa. Estos resultados también coinciden con los publicados por Untiveros (5) quien refiere que los analfabetos y los que cursaron estudios primarios alcanzan un $51,3 \%$ como prevalencia de la Diabetes Mellitus tipo 2.

Nuestros datos demuestran que la complicaciones más frecuentes fueron las infecciones, mientras que para Tapia (4), fueron las neuropatías periféricas $(87,5 \%)$ y nefropatías $(87,3 \%)$ en un estudio realizado en Hospital Cayetano Heredia en el año 1998; estas cifras son bastante mayores a nuestros hallazgos.

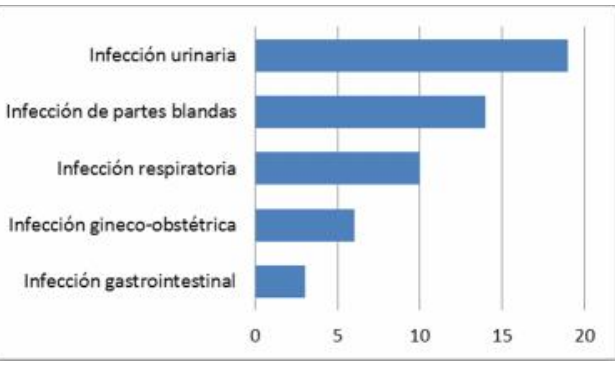

FIGURA 1. Infecciones según área de afectación en pacientes adultos mayores con Diabetes Mellitus tipo 2, hospitalizados en el Hospital Santa María del Socorro. Ica, Perú 2013

En este estudio se han observado limitaciones de orden administrativo como el que se presentó al acceder a las historias clínicas, en las que encontró datos incompletos, y en algunos casos mal llenadas y deterioradas.

Los estudios considerados como antecedentes no reportan cifras análogas a las publicadas en esta investigación, por lo constituye una limitación de orden metodológico y no permite cumplir con el principio de la comparabilidad.

Finalmente concluimos que tanto las características sociodemográficas y clínicas de la DM tipo 2 son de importancia y que deben de ser descritas y analizadas en cada región, ya que como se ha presentado están presentan una considerable variabilidad en las referencias revisadas.

\section{REFERENCIAS BIBLIOGRÁFICAS}

1. American Diabetes Association. Diagnosis and Classification of Diabetes Mellitus. Diabetes Care 2009; 32(Supp 1): S62-S67.

2. Brenes-Camacho G, Rosero Bixby L. Diabetes mellitus en adultos mayores costarricenses. Población y Salud en Mesoamérica. 2007; 5(1):1-12. [Link]

3. De la Paz Castillo KL, Proenza Fernández L, Gallardo Sánchez $\mathrm{Y}$, Fernández Pérez $\mathrm{S}$, Mompié Lastre A. Factores de riesgo en adultos mayores con diabetes mellitus. MEDISAN 2012; 16(4): 489-497. [Link]

4. Tapia Zegarra GG; Chirinos Cáceres JL, Tapia Zegarra LM. Características sociodemográficas y clínicas de los pacientes diabéticos tipo 2 con infecciones adquiridas en la comunidad admitidos en los servicios de Medicina del Hospital Nacional Cayetano Heredia. Rev Med Hered 2000; 11(3): 89-96. [Link]

5. Untiveros Mayorga CF, Nuñez Chavez O, Tapia Zegarra LM, Tapia Zegarra GG. Diabetes mellitus tipo 2 en el Hospital II Essalud Cañete: aspectos demográficos y clínicos. Rev Med Hered 2004; 15(1): 19-23. [Link] 
FINANCIAMIENTO: Autofinanciado.

AGRADECIMIENTOS: Al Hospital Santa Maria del Socorro, por permitir el estudio y al servicio de Estadistica por el acceso a las historias clinicas.

CONTRIBUCIONES DE AUTORÍA: EERM y MCGO participaron en el diseño del estudio, el análisis de los datos, revision crítica del artículo y aprobación de la versión final.

\section{Situational state of diabetes mellitus type 2 older adult patients in hospital Santa María del Socorro. Ica, Peru}

$\mathrm{n}$ order to determine the characteristics of type 2 diabetes mellitus in the elderly at the Santa Maria Hospital Relief Ica in 2013, a descriptive study was conducted, the population consisted of adult patients diagnosed with Diabetes Mellitus Type 2 treated at the Hospital Santa María del Socorro in 2013. Our findings suggest that patients $60-75$ years of age $(60 \%)$ patients in urban areas $(45 \%)$ with primary-school education (36.7\%) and married patients (38.3\%) are those in a higher proportion Diabetes Mellitus type 2, also the most frequent clinical features were urinary infection; cerebrovascular disease, and hypertension. Finally we conclude that there are many sociodemographic and clinical characteristics of importance in the management of elderly patients with type 2 diabetes mellitus.

Key words: Diabetes Mellitus, Type 2 , Aged, Population Characteristics, Diabetes Complications (source: MeSH NLM)

CITA SUGERIDA.

Ramos-Mayta EE, Gonzales-Olarte MC. Estado situacional de la diabetes mellitus tipo 2 en pacientes adultos mayores en el hospital Santa María del Socorro. Ica, Perú. Rev méd panacea.2014; 4 (3):74-76. 